\title{
SMOOTHING DEMAND BY AGGREGATION WITH RESPECT TO WEALTH
}

\author{
Egbert DIERKER, Hildegard DIERKER and Walter TROCKEL* \\ University of Bonn, Sonderfor schungshereich 21, 5300 Bonn 1, W. Germany
}

Received November 1978, accepted March 1979

\begin{abstract}
We suggest in this paper to treat the problem of smoothing demand by aggregation in a two-step procedure, corresponding to the two different constituents of consumption characteristics, wealth and preferences. Instead of imposing a manifold structure on preferences we exploit the nice structure of wealth-space. The lirst step of this procedure, aggregation with respect to wealth, is carried out. It is shown that, for any preference, aggregation with respect to wealth yields a mean demand which is almost everywhere $C^{1}$. Moreover, it is shown that for an important class of preferences, vanishing Gaussian curvature of indifference surfaces does not destroy differentiakility of the mean demand function.
\end{abstract}

\section{Introduction}

The purpose of this article is to study those phenomena which, in case of non-convex preferences, make it questionable whether demand, aggregated with respect to many consumers, is differentiable.

In our opinion it would be desirable to have a differentiable aggregate demand function for the following reasons:

Firstly, in contrast to microtheory which allows individual preferences to be non-convex, nacrotheory teaches that aggregate demand is a function and that moreover this function can be locally approximated by a linear function. Also empirical demand analysis stipulates a well behaved aggregate demand function. Therefore it would be desirable to have a microtheoretical basis for the concept of a smooth aggregate demand function.

Secondly, decentralization of consumption decisions by means of prices would hardly be possible without aggregate demand being smoother than individual demand. Non-convexity of individual preferences leads to jumps in individual demand behavior. If the consumption sector is described by an atomless measure space then gaps in the demand correspondence are automatically filled in by aggregation according to Lyapunov's theorem. There is no reason, however, why individual consumption decisions at an equilibriuin piicu system should clear the market, because the value of the aggregate demand correspondence can be a large convex set.

\footnotetext{
*The authors have benefitted from conversations with several colleagues and guests at the Sonderforschungsbereich 21 in Bonn. Financial support of the Deutsche Forschungsgemeinschaft is gratefully acknowledged.
} 
But even a continuous aggregate demand function which may have extremely steep slopes is not suitable to explain the decentralization of demand decisions. For it is well known that the graph of a convex-valued upper-hemicontinuous (u.h.c.) demand correspondence can be approximated by the graph of a continuous function. The slope of the approximating function becomes extremely steep close to points whose image sets under the correspondence are not singletons. Thus, a continuous function may be hardly distinguishable from a correspondence. Therefore it is essential that the slopes of the aggregate demand function are bounded near equilibrium prices. Otherwise, if demand were too sensitive with respect to prices, very small price variations could lead to a considerable deviation from equilibrium.

Thirdly, much of the explanatory value of equilibrium theory is based on the concept of a regular equilibrium. The application of this concept to an economy with a measure space of consumers requires differentiability of the mean demand function. In this context we like to quote Debreu (1972, p. 614): 'One expects that, if the measure $v$ is suitably diffused over the space $A$, integration over $A$ of the demand correspondences of the agents will yield a to'al demand function. possibly even a total demand function of class $C^{1}$.'

Lintil now we ! ave been arguing from an economic point of view why we think thic concept of a $C^{1}$ mean demand function to be a useful one. Maybe from a mathemitician's point of view it would be more natural to treat the problem in a $C^{\prime}$ setting. From corrinents by $\mathrm{R}$. Tliom on the problem of obtaining a $C^{\prime}$ mean demand function we concluded that this is a very hard problem requiring deep insights into singularity theory. Furthermore, for the application of singularity theory to this problem, a smooth manifold structure on the space of preferences is needed, a structure which we do not want to impose:

The paper closest related to ours is Sondermann (1976). His and our intention is to state conditions under which mean demand becomes $C^{1}$. Given the present state of the subject these conditions need not be generic. Sondermann's approach involves a smooth manifold structure on the space of preferences and relies on the study of catastrophes. He pointed out that a major difficulty in the analysis of the problem arises from the fact that jumps of the individual demand, which occur at a given price system for a null set of consumers. do affect the derivative of the mean demand function. In our approach we essentially take care of the influence of these null sets by aggregating demand with respect to wealth, keeping preferences fixed. We intend to aggregate with respect to preferences by interchanging differentiation and integration using Lebesgue's Dominated Convergence Theorem.

For a discussion of Sondermann's approach the reader is referred to Araujo and Mas-Colell (1978). Their paper also contains a short proof of a 
theorem due to Sondermann (1975) yielding the continuity of the mean demand function.

To motivate the idea that aggregation should be carried out in two steps, first with respect to wealth, then with respect to preferences, it is useful to look at the extreme case where all goods are indivisible. To make things simple assume that there are two commodity bundles only, $x$ and $y$, between 'vhich consumers are not indifferent. There are only two possible preference relations. Consider the class of consumers preferring $x$ to $y$. The mean demand of this class of consumers is a convex combination of $x$ and $y$ with weights depending on prices and wealth. Assume that the wealth distribution of this class of consumers has a continuous density with respect to the Lebesgue measure. Then the weights in the convex combination of $x$ and $y$ vary differentiably with prices except on that closed null set of prices which assign the same value to $x$ and $y$. Therefore, considering a fixed preference relation, one obtains by aggregation with respect to a suitable wealth distribution a mean demand function which is $C^{1}$ except on a subset of codimension one in the set of prices. The derivative of this mean demand function is determined by those consumers who can just afford to switch from $y$ to $x$. The first step of the aggregation procedure is an analogue of this idea in the framework of perfectly divisible commodities. This step is carried out in the present article. For each preference relation it brings about a considerable smoothing effect.

Suppose now that there are very many commodity bundles and. accordingly, very many preference relations. For each preference relation there is a null set of prices where the corresponding mean demand may have a kink. If, at a given price system, a kink occurs only for a inearly negligible set of consumers then these kinks play almost no role for the bihavior of the mean demand of the whole consumption sector. One may hope to get rid of the non-differentiability remaining after aggregation with respect to wealth by aggregation with respect to preferences. It is essential for the second step that preferences are dispersed.

The analysis in the perfectly divisible case is not quite as simple as suggested by the above sketchy remarks. In this paper we intend to show which kind of obstacles for differentiability uccur. We will fix a not necessarily convex preference and investigate which amount of smoothness of demand can be obtained by aggregation with respect to wealth. We will see that this first step of aggregation yields a demand which is a $C^{1}$ function except on a closed null set ur prices, depending on the given preference relation. $^{1}$

The prices in this exceptional null set correspond to three types of

\footnotetext{
${ }^{1}$ For prices in this exceptional set demand need not be unique. In another paper [DierkerDierker Trockel (1980)] we show that for most preferences aggregation with respect to wealth yields a continuous mean demand function.
} 
difficulties: vanishing Gaussian curvature of indifference surfaces, critical simple jumps and non-critical multiple jumps. A simple resp. multiple jump at a price $p$ denotes a situation where the cardinality of the demand set at $p$ is two resp. greater than two. A jump is called critical if there are points in the demand set of $p$ with equal marginal utilities.

The first difficulty leads to the study of unfoldings of degenerate germs. Restricting ourselves to the simplest important case, the cusp catastrophe, we show that vanishing Gaussian curvature does not destroy differentiability of mean demand for a fixed preference, as long as no other disturbance occurs simultaneously and some rank condition is fulfilled.

Critical simple jumps, to the contrary, require a good deal of dispersedness of preferences.

Multiple (non-critical) jumps do not destroy the differentiability of mean demand, provided that they occur, at any given price system, for a null set of preferences only.

Aggregation with respect to preferences in order to treat critical and multiple jumps will not be carried out in this paper.

\section{Description of the model}

The consumption set for every consumer is

$$
X:=\left\{x \in \boldsymbol{R}^{l} \mid x \gg 0\right\} .
$$

We consider prices in

$$
S:=\left\{p \in \boldsymbol{R}^{l}\left|p \gg 0,\|p\|=\sum_{i=1}^{l}\right| p_{i} \mid=1\right\} .
$$

An individual agent is described by his wealth $w$ and by his preference $\lesssim$. The wealth space is assumed to be the compact interval $[\underline{w}, \bar{w}] \subset \mathscr{\boldsymbol { R }}_{+}, \underline{w}<\overline{\bar{w}}$. For any set $\hat{\mathscr{P}}$ of utility functions on $X$ two elements $u_{1}, u_{2} \in \hat{\mathscr{P}}$ are considered equivalent $\left(u_{1} \sim u_{2}\right)$, if for all pairs of commodity bundles $x, y \in X$,

$$
u_{1}(x)>u_{1}(y) \Leftrightarrow u_{2}(x)>u_{2}(y) .
$$

In the following we consider the quotient space $\mathscr{P}:=\hat{\mathscr{P}} / \sim$ where $\hat{\mathscr{P}}$ is the set of all $C^{x}$ utility functions fulfilling $\mathscr{U} 1, \mathscr{U} 2, \mathscr{U} 3$ below. $\mathscr{P}$ is the space of all preference orderings that are representable by $C^{\gamma}$ utility functions in $\hat{\mathscr{P}}$.

$$
\text { III: } D u(x) \gg 0 \text { for all } x \in X \text { (monotonicity). }
$$


Let $g(x):=D u(x) \cdot\|D u(x)\|^{-1}$. The following assumption keeps demand away from the boundary of the consumption set:

$\mathscr{U} 2$ : For any $x \in X$, the set $\mathrm{cl}^{-1}(u(x))$ is contained in $X$.

The fact that $g^{-1}(p)$ is a smooth one-dimensional manifold "varying smoothly' with $p$ is very helpful for showing that mean demand is $C^{1}$. We therefore 'ssume:

刃3: $g: X \rightarrow S$ has full rank $l-1$ everywhere.

This assumption allow's indifference hypersurfaces to have Gaussian curvature zero. However, it precludes two or more principal curvatures to vanish simultaneously.

For Theorem 1 we need two more assumptions on preferences:

Assumption $\mathscr{U} 4$ is made to handle the case of indifference surfaces with vanishing Gaussian curvature, $k$. The concept of Gaussian curvature has been used by Debreu (1972) in order to study the differentiability of demand functions derived from preferences. Assume $k(\tilde{x})=0$ and let $\tilde{p}=g(\tilde{x}), \tilde{w}=\tilde{p} \tilde{x}$. Denote the restriction of the utility function $u$ to the budget hyperplane $\{x \in X \mid p x=w\}$ by $u_{\mid p, \ldots .}$ The family $\left\{u_{\mid p, w} \mid(p, w) \in S \times \dot{\boldsymbol{R}}_{+}\right\}$can be regarded as an $l$-parametric unfolding of $u_{\mid p . w^{\prime}}$. More precisely, let $U: \hat{\boldsymbol{R}}_{+}^{l-1} \times S \times \hat{\boldsymbol{R}}_{+} \rightarrow \boldsymbol{R}$ bc defined by

$$
U\left(x_{1}, \ldots, x_{l-1}, p \cdot w\right):=u\left(x_{1}, \ldots, x_{l-1}, x_{l}(p, w)\right)
$$

where

$$
x_{l}(p, w)=\left(w-\sum_{i=1}^{l-1} p_{i} x_{i}\right)\left(1-\sum_{i=1}^{l-1} p_{i}\right)^{-1} .
$$

We write $U(\cdot, \tilde{p}, \tilde{w}): \dot{\boldsymbol{R}}_{+}^{l-1} \rightarrow \boldsymbol{R}$ instead of $u_{\mid \dot{\boldsymbol{p}}, \tilde{w}}$ and consider the $l$-parametric unfolding $U$ of the germ represented by $U(\cdot, \tilde{p}, \tilde{w})$ at $\hat{\tilde{x}}=\left(\tilde{x}_{1}, \ldots, \tilde{x}_{l-1}\right)$. It is natural to concentrate on the class of unfoldings of the singularity $U(\cdot, \tilde{p}, \bar{i})$ at $\hat{\tilde{x}}$ having the property that nearby unfoldings represent the same qualitative behavior. These unfoldings are the stable ones. A stable unfolding enjoys the property that it represents all the 'deformations' of the singularity at $(\tilde{p}, \tilde{w})$ for suitable parameters $p, w$. We therefore assume:

II4: The unfolding $U$ is stable.

Stability of unfoldings is defined in various equivalent ways in Wassermann (1974, \$4). Assuming that $U$ is stable amounts to requiring $U$ to be versal in Bröcker's (1975) terminology. 
The next assumption, $\mathscr{U} 5$, is made to rule out preferences with a somewhat arificial properiy. Loosely speaking, we want to exclude preferences exhibiting triples of commodity bundles in a demand set which are stable with respect to infinitesimal price variation in all directions. For $l=2$, the phenomenon to be excluded is an infinitesimal version of a situation such as:

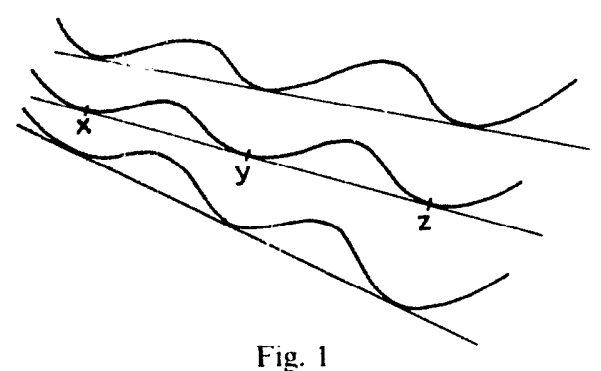

Fig. 1

More precisely, assume that three pairwise distinct commodity bundles $x . y: z$ are demanded at price $p$ and wealth $w$, i.e., the bundles $x, y, z$ are $\lesssim$ maximai in $\left.; x^{\prime} \in X \mid p x^{\prime} \leqq w\right\}$. Then $u(x)=u(y)=u(z)$ and $p x=p y=p z$. Furthermore, $D u(x), D u(y)$, and $D u(z)$ are proportional to $p$. Hence there exist numbers $x, \beta, ;$ such that $p=x D u(x)=\beta D u(y)=\gamma D u(z)$. We want to exclude the case where $x-y=[(x-\beta) /(\beta-\gamma)] \cdot(y-z)$.

Counting equations characterizing the exceptional triples one sees that there are $3 l+1$ restrictions. As there are only $3 l$ degrees of freedom, it is plausible that only 'few' preferences exhibit triples $(x, y, z)$ of the kind to be excluded. We assume

$$
\begin{aligned}
& \text { 115: Let }(x, y, z) \in X \times X \times X, \quad x \neq y, \quad x \neq z, \quad y \neq z . \\
& \text { Assume } u(x)=u(y)=u(z), \\
& \alpha D u(x)=\beta D u(y)=i D u(z)=p . \\
& p x=p y=p z \text { and } x-y=i(y-z) . \\
& \text { Then } i \neq(x-\beta) /(\beta-\eta) .
\end{aligned}
$$

We endow the space $\hat{P}$ of $C^{\varkappa}$ utility functions fulfilling $\mathscr{U}, \mathbb{\ell}, \mathbb{U} 3$ with the topology of uniform $C^{x}$ convergence of functions on compact sets. This is a metrizable separable topology. The space $\mathscr{P}=\hat{\mathscr{P}} / \sim$ with the identification topology determined by the projection $\pi: \hat{\mathscr{P}} \rightarrow \hat{\mathscr{P}} / \sim$ is the space of preferences. According to Mas-Colell (1977, p. 1391) the same metrizable separable topology on $\mathscr{P}$ can be established in the following way. Associate the mapping $g: X \rightarrow S$ defined by $: r \mapsto D u(x) \cdot\|D u(x)\|^{-1}$ with any preference $\lesssim$ in $\mathscr{P}$, where $u \in \hat{P}$ represents $\lesssim$. Endow the set of these mappings $g$ with the topology of uniform $C^{x}$ convergence on compact sets. 
The demand set of an agent with preference $\lesssim \in \mathscr{P}$ and wealth $w \in[\underline{w}, \bar{i}]$ at the price system $p \in S$ is

$$
\varphi(\lesssim, p, w)=\{x \in X \mid p x \leqq w, x<y \Rightarrow p y>w\}
$$

The demand correspondence $\varphi: P \times S \times[\underline{w}, \bar{w}] \rightarrow X$, defined in this way, is upper-hemicontinuous (u.h.c.) [Mas-Colell (1977, p. 1391)]. For any $p \in S$ this defines a u.h.c. correspondence $\varphi(\cdot, p, \cdot): \mathscr{P} \times[\underline{w}, \bar{w}] \rightarrow X$ which has a measurable graph [Hildenbrand $(1974$, p. 102)]. To define aggregate demand we need a measure on the space $\mathscr{P} \times[\underline{w}, \bar{w}]$ o. consumers' characteristics. The smoothing effect of aggregation can be expected only if consumers' characteristics are dispersed enough. One is tempted here to speak of "equally distributed' characteristics. However, it is by no means slear what 'equal distribution' might be unless one considers spaces where Leoesgue (or at least Haar) measure is available. It seems to us that the space of preferences, as far as we understand it today, has too little structure for the distinction of a natural class of suitably diffused measures. Therefore we shall exploit the fact that the wealth space is a subset of the real line.

For any topological space $T$, let $\mathscr{B}(T)$ denote the Borel $\sigma$-field of $T$. We assume

$\mathscr{H}$ : For any preference $\lesssim \in \mathscr{P}$ there is a probability measure $\delta$ : on $([\underline{w}, \bar{w}], \mathscr{B}[\underline{w}, \bar{w}])$ with a continuous density. $h_{-}$, with respect to the Lebesgue measure $i$ such that $h_{z}(\underline{u})=h_{z}(\bar{i})$ $=0$.

We do not need any measure on the space of preferences in this paper since we consider only one fixed preference in $\mathscr{P}$. But, for any probability $\mu$ on $(\mathscr{P}, \mathscr{B}(\mathscr{P}))$ assumption $\mathscr{U}$ is consistent with the existence of a probability $\tau$ on $(\mathscr{P} \times[\underline{w}, \bar{w}], \mathscr{B}(\mathscr{P} \times[\underline{w}, \bar{w}]))$ such that $\mu$ is the marginal distribution of $\tau$ on $(\mathscr{P}, \mathscr{B}(\mathscr{P}))$, and $\delta: \mathscr{P} \times \mathscr{B}([\underline{w}, \bar{w}]) \rightarrow[0,1]$, with $\delta(\lesssim, \cdot)=\delta_{\preccurlyeq}$, is a regular conditional $\tau$-distribution for $(\lesssim, w) \mapsto w$ given $(\lesssim, w) \mapsto \lesssim$. To see this we define $h: \mathscr{P} \times[\underline{w}, \bar{w}] \mapsto \boldsymbol{R}$ in such a way that for any $\lesssim \in \mathscr{P}$ and for any $w \in[\underline{w}, \bar{w}]$ the function $h(\lesssim, \cdot):[\underline{w}, \bar{w}] \rightarrow \boldsymbol{R}$ is continuous and the function $h(\cdot, w): \mathscr{P} \rightarrow \boldsymbol{R}$ is $\not{B}[\underline{w}, \bar{w}]$-measurable. Then $h$ is a $\not \mathscr{B}(\mathscr{P} \times[\underline{w}, w])$-measurable function [Hildenbrand (1974)]. Now $\int_{M} h(\lesssim, w) \lambda(\mathrm{d} w)=\delta(\lesssim, M)$ for any $M \in \mathscr{B}([\underline{w}, \bar{w}])$ defines a transition probability $\delta: \mathscr{P} \times \mathscr{B}([\underline{w}, \bar{w}]) \rightarrow[0,1]$. Then a unique probability $\tau$ on $(\mathscr{P} \times[\underline{w}, \bar{w}], \mathscr{B}(\mathscr{P} \times[\underline{w}, \bar{w}]))$ is defined by [Friedman (1971, pp. 347, 348)]

$$
\tau(Q)=\int_{\mathscr{P}} \delta\left(\lesssim, Q_{\lessgtr}\right) \mu(\mathrm{d} \lesssim) \quad \text { for any } \quad Q \in \mathscr{B}(\mathscr{P} \times[\underline{w}, \bar{w}])
$$


The set $Q_{\S} \in \mathscr{B}([\underline{w}, \bar{w}])$ is the $\lesssim$-section of $Q$. The mean demand formula then reads as

$$
\begin{aligned}
& F(p)=\int_{p \times[u, \bar{w}]} \varphi(\lesssim, p, w) \mathrm{d} \tau \\
& =\int_{\mathscr{P}} \int_{\underline{\underline{w}}}^{\bar{w}} \varphi\left(\precsim, p, w^{\prime}\right) \delta(\lesssim, \mathrm{d} w) \mu(\mathrm{d} \precsim) \\
& =\int_{\mathscr{P}} \int_{w}^{\bar{w}} \varphi(\lesssim, p, w) h_{\S}(w) \hat{\lambda}(\mathrm{d} w) \mu(\mathrm{d} \lesssim) \\
& =\int_{\phi} \Phi(\lesssim, p) \mu(\mathrm{d} \lesssim)
\end{aligned}
$$

For any $\lesssim \in \mathscr{P}$ the function $h_{\S}=h(\precsim, \cdot)$ is the continuous density of $\delta(\precsim, \cdot)$ $=\delta \lesssim$ with respect to the Lebesgue measure $\lambda$ on $([\underline{w}, \bar{w}], \mathscr{B}([\underline{w}, \bar{w}]))$.

In this paper we shall analyze the smootheness properties of $\varphi(\lesssim, \cdot)$ for any fixed preference $\lesssim \in \mathscr{P}$.

\section{Aggregation with respect to wealth}

In this section we shall prove the following result:

Theorem 1. Let $\lesssim \in \mathscr{P}$. Under the assumptions $\mathbb{M} 4, \mathbb{M} 5$ and $\mathscr{M}$ there is a closed null set $N_{\leqslant}$in the price space $S$ such that the restriction to $S \backslash N_{\leqslant}$of $\Phi(\lesssim, \cdot)=\int_{w}^{i} \varphi(\lesssim, \cdot, w) h_{\S}(w) \lambda(\mathrm{d} w)$ is a $C^{1}$ function.

To prove the theorem we look at three phenomena which may destroy the differentiability of $\Phi(\lesssim, \cdot)$, and we show that each of these phenomena can occur on a small set only.

To simplify notation we will drop the symbol $\lesssim$ in $\varphi(\cdot)$ in the rest of the paper since the preference is fixed. Moreover we drop the density $h_{\S}$ occasionally as this does not change the arguments.

The first disturbing phenomenon is the vanishing Gaussian curvature.

Let $X_{0}=\left\{x \in Y|k(x)=0, u|_{g(x), g(x) x}\right.$ has a maximum at $\left.x, \underline{w} \leqq g(x) x \leqq \bar{w}\right\}$.

Lemma 1. Assume $\mathbb{M} 4$. Then $N_{0}=g\left(X_{0}\right)$ is a closed mull set.

Proof. Let $C$ be any compact subset of $S$. To show that $N_{0}$ is closed it suffices to show the closedness of $N_{0} \in C$. Consider $X_{C}:=\{x \in X \mid g(x) \in C$, $\underline{w} \leqq g(x) x \leqq \bar{w}\}$. Obviously $X_{C}$ is bounded and closed in $X$. Assumption $\mathscr{U} 2$ implies that $X_{\mathrm{C}}$ stays away from the boundary $\bar{X} \backslash X$. Therefore, $X_{\mathrm{C}}$ is a compact subset of $X$. As $X_{0}$ is closed, $X_{0} \cap X_{C}$ and hence $N_{0} \cap C$ $=g\left(X_{0} \cap X_{C}\right)$ is compact. 
Now we show that $N_{0}$ has zero measure. Let $X_{0}^{r}$ denote the set of points $\tilde{x} \in X_{0}$ such that $\left.u\right|_{g(\hat{x}), g(\hat{x}) \dot{x}}$ has a singularity of codimension $r$ at $\tilde{x}$. We have $r \geqq 2$ since $\left.u\right|_{g(\tilde{x}), g(\hat{x}) \dot{x}}$ has a local maximum at $\tilde{x}$, and $r \leqq l$ because of $\mathscr{U} 4$. It suffices to show that $g\left(X_{0}^{r}\right)$ has measure zero for any $r \in N, 2 \leqq r \leqq l$. The unfolding $U$ of assumption $\dddot{U} 4$ is equivalent to the sum of a universal unfolding $f_{u}$ with $r$ parameters and a constant unfolding $f_{c}$ with $l-r$ parameters [Bröcker (1975, p. $123 \mathrm{f}$.)].

If we can show for the universal unfolding that, in a neighborhood of any singularity of codim $r$, only singularitics with codim $<r$ can occur, we know that the unfolding $U$ can have singularities of codim $r$ near $(\tilde{x}, g(\tilde{x}), g(\tilde{x}) \tilde{x})$ only on an $(l-r)$-dimensional manifold. The projection $(p, w) \mapsto p$ takes this $(l-r)$-dimensional manifold into a null set, because $r \geqq 2$. If follows that $g\left(X_{0}^{r}\right)$ is a null set.

Therefore it remains to show that for the universal $r$-parameter unfolding $f_{u}$ of a singularity with codim $r$ the following is true: For any parameter $v \in \boldsymbol{R}^{\mathbf{r}}$ in a neighborhood of zero the mapping $f_{u}(\cdot, v)$ has only singularities of codim $<r$. Since, by $\mathscr{U} 3$, the singularity $U(\cdot, g(\tilde{x}), g(\tilde{x}) \tilde{x})$ at $\hat{\tilde{x}}$ has corank 1 . the germ of $f_{v}$ at $\hat{\tilde{x}}$ can be transformed into the equivalent germ $\eta:(\boldsymbol{R}, 0)$ $\rightarrow(\boldsymbol{R}, 0)$ given by $x \mapsto x^{r+2}$. According to Bröcker $(1975$, p. 124) we can choose the universal unfolding of $\eta$ to have the following form:

$$
f_{u}(x, v)=x^{r+2}+v_{r} x^{r}+\cdots+v_{1} x
$$

Now, according to Bröcker $\left(1975\right.$, p. 123), codim $\eta=\operatorname{dim}_{R}(m(1)$ $\left.\langle\hat{\partial} \eta / \hat{r} x\rangle_{\delta(1)}\right)$. The set $\mathscr{E}(1)$ denotes the smooth germs $(\boldsymbol{R}, 0) \rightarrow \boldsymbol{R}$, and $m(1)$ denotes the smooth germs $(R, 0) \rightarrow(R, 0)$.

By some computation one gets that for $\left(v_{1}, \ldots, v_{r}\right) \neq 0$ the ideal $\left\langle\partial f_{u}(\cdot, v) / \partial x\right\rangle_{\delta(1)}$ has smaller codimension than the ideal $\langle\hat{c} \eta / \hat{c} x\rangle_{\delta(1)}$. Therefore codim $f_{u}(\cdot, v)<r$. Q.E.D.

In order to avoid the case of vanishing Gaussian curvature we concentrate upon prices in $S_{1}=S \backslash N_{0}$. We are now going to study pairs of commodity bundles which may belong to the same demand set $\varphi_{\S}\left(p, w^{\prime}\right)=\varphi\left(\lesssim, p, w^{\prime}\right)$.

For that purpose let

$$
X_{1}=\left\{x \in X \mid \text { Hessian of }\left.u\right|_{\operatorname{Ker} D u x} \text { is negative definite }\right\}
$$

be the set of commodity bundles where preferences are locally convex and have non-zero Gaussian curvature. The diagonal of $X_{1} \times X_{1}$ is denoted $\Delta_{1}$. We employ the symbol $\wedge$ in order to delete the last component of a vector in $\boldsymbol{R}^{\boldsymbol{l}}$. 


\section{Lemma 2. The mapping}

$$
G_{1}: S_{1} \times\left(X_{1} \times X_{1}: \Delta_{1}\right) \rightarrow R^{1-1} \times R \times R^{l-1} \times R,
$$

defined by

$$
G_{1}(p, x, y)=(\hat{g}(x)-\hat{p}, u(x)-u(y), \hat{g}(y)-\hat{p}, p(x-y)),
$$

has zero as a regular value.

Proof. The derivative $D G_{1}(p, x, y)$ can be represented by the following matrix:

$$
\left(\begin{array}{ccc}
-I_{(l-1, l-1)} & \frac{\partial g_{i}}{\partial x_{j}}(x)\left(\begin{array}{c}
i=1, \ldots, l-1 \\
j=1, \ldots, l
\end{array}\right) & 0 \\
0 & D u(x) & -D u(y) \\
-I_{(l-1, l-1)} & 0 & \frac{\partial g_{i}}{\partial y_{j}}(y) \\
\hat{x}-\hat{y}-(x-y)_{l} \hat{e} & p & \left(\begin{array}{c}
i=1, \ldots, l-1 \\
j=1, \ldots, l
\end{array}\right) \\
-p
\end{array}\right)
$$

where $\hat{e}=(1, \ldots, 1) \in R^{l-1}$ and $(x-y)_{l}$ is the last component of $x-y$. From Debreu (1972, p. 28) we know that

$$
\left(\begin{array}{l}
\frac{\partial g_{i}}{\partial y_{i}}(y) \\
D u(y)
\end{array}\right) \text { has rank } l \text { because } k(y) \neq 0
$$

It remains to show that

$$
\left(\begin{array}{cc}
-I & \frac{\partial g_{i}}{\partial x_{j}}(x) \\
\hat{x}-\hat{y}-(x-y)_{l} \hat{e} & (c x-\beta) D u(x)
\end{array}\right)
$$

has rank $l$, where $\alpha D u(x)=p, \beta D u(y)=p$. If $\alpha \neq \beta$, then the rank is $l$ because $\kappa(x) \neq 0$. If $\alpha=\beta$, then the raik is $l$ provided $\hat{x}-\hat{y} \neq(x-y)_{l} \hat{e}$. This inequality, however, follows from monotonicity if $u(x)=u(y)$. Q.E.D.

Corollary. $\Gamma_{1}=G_{1}^{-1}(0)$ is a $C^{1}$ manifold of dimension $1-1$.

Let $N_{1}$ be the set of critical values of $\left.\pi_{1}\right|_{\Gamma_{1} \cap\left(S_{1} \times\left(X^{\prime} \times X^{n}, \Delta\right)\right.}$ where $\pi_{1}$ $=\left.\operatorname{proj}\right|_{\Gamma_{1}}: \Gamma_{1} \rightarrow S_{1}$ and $X^{\prime}=\{x \in X \mid \underline{w} \leqq g(x) \cdot x \leqq \bar{w}\}$ and $\Delta$ is the diagonal of $X^{\prime} \times X^{\prime}$. 
Lemma 3. $N_{1}$ is closed in $S_{1}$ and null. A price system $p_{1}$ is in $N_{1}$ if there is $\left(p_{1}, x, y\right) \in \pi_{1}\left(p_{1}\right)$ such that $D u(x)=D u(y)$.

Proof. A necessary and sufficient condition for $p_{1}$ to be a critica! value of $\pi_{1}$ is that there is $\left(p_{1}, x, y\right) \in \Gamma_{1}$ such that $G_{1}\left(p_{1}, \cdot, \cdot\right)$ has less than full rank at $(x, y)$. A calculation similar to the one above yields that this happens iff $D u(x)$ $=D u(y)$.

Let $C$ be a compact subset of $S_{1}$. As in the proof of Lemma 1 it suffices to show that $N_{1} \cap C$ is a closed null set. Let $X_{C}=\{x \in X \mid g(x) \in C, w \leqq g(x) \cdot x$ $\leqq \bar{w}$. We know that $X_{C}$ is compact and claim that $\Gamma_{1} \cap\left[C \times\left(X_{C} \times X_{C} \backslash \Delta_{C}\right)\right]$ is compact. $\Delta_{C}$ denotes the diagonal of $X_{C} \times X_{C}$.

To proof this assertion, consider a sequence $\left(p_{n}, x_{n}, y_{n}\right)_{n=1,2 \ldots}$ of points in $\Gamma_{1} \cap\left[C \times\left(X_{C} \times X_{C} \Delta_{C}\right)\right]$ converging to $\left(p_{10}, x_{0}, y_{0}\right) \in C \times X_{C} \times X_{C}$. We have to show that $x_{0} \neq y_{0}$.

Assume the contrary. Without restriction we can assume that the sequence $\left(x_{n}-y_{n}\right) /\left\|x_{n}-y_{n}\right\|$ of elements of the $(i-1)$-sphere converges to a vector $z$. The directional derivative of $g$ at $x_{0}$ in the direction $z$ must vanish because $g\left(x_{n}\right)-g\left(y_{n}\right)=0$ for all $n$. This contradicts the assumption that the Hessic." of $\left.u\right|_{\operatorname{Ker} D u(x)}$ at $x_{0}$ is negative definite.

According to Sard's theorem the set of critical values of $\pi_{1} \mid I_{1} r\left[C \times 1 X_{C} \times Y_{\left.\left(1, H_{(}\right)\right]}\right.$ is a closed null set. Q.E.D.

Next we examine the case of three commodity bundles which may belong to the same demand set $\varphi_{\S}(p, w)$.

However, we shall show that this case can occur for a small set of prices only. To exclude the disturbing phenomena we dealt with before we concentrate upon prices in $S_{2}=S_{1} \backslash N_{1}$.

Let $E=\left\{(x, y, z) \in X_{1} \times X_{1} \times X_{1} \mid x=y\right.$ or $x=z$ or $\left.y=z\right\}$.

\section{Lemma 4. The mapping}

$$
G_{2}: S_{2} \times\left[\left(X_{1} \times X_{1} \times X_{1}\right) \backslash E\right] \rightarrow R^{l-1} \times R \times R^{l-1} \times R \times R^{l-1} \times R \times R,
$$

defined by

$$
\begin{aligned}
G_{2}(p, x, y, z)= & (\hat{g}(x)-\hat{p}, u(x)-u(y), \hat{g}(y)-\hat{p}, u(y) \\
& -u(z), \hat{g}(z)-p, \hat{p}(x-y), p(y-z)),
\end{aligned}
$$

has zero as a regular value.

Proof. The proof is similar to that of Lemma 2. One represents 
$D G_{2}(p, x, y, z)$ by a matrix and finds that this matrix has rank $3 l+1$ provided

$$
(\hat{y}-\hat{z})-(y-z)_{l} \hat{e} \neq \frac{\alpha-\beta}{\beta-\hat{\gamma}}\left[\hat{x}-\hat{y}-(x-y)_{l} \cdot \hat{e}\right] .
$$

But this inequality holds by assumption $\dddot{\text { L. }} \quad$ Q.E.D.

Corollary: $\quad \Gamma_{2}=G_{2}^{-} \cdot(0)$ is a $C^{1}$ manifold of dimension $l-2$.

Lemma 5. There is a null set $N_{2}$ of price systems closed in $S_{2}$ such that for any $p \in S_{2} \backslash N_{2}$ and any $w \in[w, \bar{w}]$ the demand set $\varphi(p, w)$ does not contain more than two elements.

Proof. Let $\pi_{2}=$ proj $\left.\right|_{\Gamma_{2}}: \Gamma_{2} \rightarrow S_{2}$. Because $\operatorname{dim} \Gamma_{2}=1-2$ all points in $\Gamma_{2}$ are critical points of $\pi_{2}$. Define $N_{2}=\pi_{2}\left(\Gamma_{2} \cap\left(S_{2} \times X^{\prime} \times X^{\prime} \times X^{\prime}\right)\right)$. By Sard's theorem and a reasoning similar to that in Lemma 3 we get that $N_{2}$ is a null set closed in $S_{2}$. Q.E.D.

\section{Pioof of Theorem 1}

Let $N_{\leqslant}:=N_{0} \cup N_{1} \cup N_{2}$ and $p_{0} \in S \backslash N_{\lessgtr}$. Hence $p_{0}$ is a regular value of $\pi_{1}$. Therefore, and by the compactness of $[\underline{w}, \bar{w}]$ there is a finite number of $C^{1}$ functions (id, $\left.x_{i}(\cdot), y_{i}(\cdot)\right)$, defined in a neighborhood $V$ of $p_{0}$ and taking values in $\Gamma_{1}$, having the following property: For any $p$ in this neighborhood $V$ of $p_{0}$ the correspondence $\varphi(p, \cdot)$ is single-valued at any $w \in] w_{i}(p), w_{i+1}(p)\left[\right.$, where $w_{i}(p)=p x_{i}(p)=p y_{i}(p)$ and $w_{i+1}(p)=p x_{i+1}(p)$ $=p y_{i+1}(p)$. Therefore we have finitely many, say $k$, smooth functions $w_{i}$ from $V$ into $[\underline{w}, \bar{w}]$. For any $p \in V$ and any $i, j \in\{1, \ldots, k\}, i \neq j$, we have $w_{i}(p)$ $\neq w_{j}(p)$. Denote by $\varphi^{i}(\cdot, \cdot)$ the inverse of the mapping $x \mapsto(g(x), g(x) x)$ restricted to a suitable neighborhood of either $x_{i}\left(p_{0}\right)$ or $y_{i}\left(p_{0}\right)$. Define the constant functions $w_{0}$ and $w_{k+1}$ by $w_{0}(p)=\underline{w}$ and $w_{k+1}(p)=\bar{w}$, respectively. Now, for $p \in V$ and for any $i \in\{0, \ldots, k\}$ and any $w \in] w_{i}(p), w_{i+1}(p)[$ we have $\varphi^{i}(p, w)=\varphi(p, w)$ and therefore

$$
\left.\int_{w_{i}(p)}^{w_{i}+1(p)} \varphi ! p, w\right) h(w) \mathrm{d} w=\int_{w_{i}(p)}^{w_{i}+1(p)} \varphi^{i}(p, w) h(w) \mathrm{d} w
$$

For the mean demand we have

$$
\Phi(\lesssim, p)=\sum_{i=0}^{k+1} \int_{w_{i}(p !}^{w_{i+1}(p)} \varphi_{\lessgtr}^{i}(p, w) h(w) \mathrm{d} w .
$$


A version of Leibniz's rule shows that this integral is $C^{1}$ at $p_{0}$ [Dieudonné (1960, p. 173)]. The derivative at $p_{0}$ of such an integral is given by the linear mapping from $\boldsymbol{R}^{l}$ in $\boldsymbol{R}^{l}$ defined by

$$
\begin{aligned}
z \mapsto\left[\int_{w_{i}\left(p_{0}\right)}^{w_{i+1}(p 0)}\right. & D_{p} \varphi^{i}\left(p_{0}, w\right) h(w) \mathrm{d} w \\
& +\varphi^{i}\left(p_{0}, w_{i+1}\left(p_{0}\right)\right) h\left(w_{i+1}\left(p_{0}\right)\right) D_{p} w_{i+1}\left(p_{0}\right) \\
& \left.-\varphi^{i}\left(p_{0}, w_{i},\left(p_{0}\right)\right) h\left(w_{i}\left(p_{0}\right)\right) D_{p} w_{i}\left(p_{0}\right)\right] z .
\end{aligned}
$$

Therefore the derivative of $\Phi(\lesssim, \cdot)$ is uniquely determined at $p_{0}$ and can be computed as stated above. Q.E.D.

Remark. The proof shows that the derivative of mean demand $\Phi(\lesssim, \cdot)$ consists of two parts. The first one describes the local substitution, the second one the jumping between commodity bundles'. The jumping effect is measured by a sum of terms of the following kind:

$$
\left(x_{i}\left(p_{0}\right)-y_{i}\left(p_{0}\right)\right) h\left(w_{i}\left(p_{0}\right)\right) D_{p} w_{i}\left(p_{0}\right)
$$

\section{Vanishing Gaussian curvature: The cusp}

In this section we want to analyze the most important and at the same time the simplest type of a situation where a commodity bundle $\tilde{x}$ with vanishing Gaussian curvature is demanded at the price system $\dot{p}=g(\tilde{x})$ and the wealth $\tilde{w}=\tilde{p} . \tilde{x}$. Since by assumption $\geqslant / 3$ the mapping $g$ has full rank at $\tilde{x}$ there is exactly one direction at $\tilde{x}$ in which the indifference surface $u^{-1}(u(\tilde{x}))$ has vanishing principal curvature. That is to say that the corank of $\left.u\right|_{p, i}$ at $\bar{x}$ is one.

Here and in the following we will not distinguish germs of maps or sets from their representatives, unless we encounter possible confusion. In the whole section we are interested only in germs. The only exception is at the end where we shall integrate functions.

In Thom's list classifying elementary catastrophes there are several catastrophes with corank one. The simplest one, the fold, belongs to a potential function such as $y \mapsto y^{3}$. As $\tilde{x}$ is demanded at price $\tilde{p}$ and wcalth $\bar{w}$ we know that $\left.u\right|_{\hat{p}, \hat{w}}$ has a local maximum at $\tilde{x}$. Hence the situation must be somewhat more complicated than a fold.

So we are led to study the next candidate of Thom's list, the cusp. In case of a cusp the utility decreases in the direction of vanishing principal curvature as $y \mapsto-y^{4}$ decreases near zero. Useful illustrations of the cusp catastrophe can be found in Bröcker (1975, pp. 148-150). In the following we shall study the question of how the cusp phenomenon affects the 
differentiability of mean demand. Since a budget hyperplane is $(l-1)$ dimensional, $\left.u\right|_{\hat{p} .1,}$ can be conceived of as $\left(y_{1}, \ldots y_{1-1}\right) \mapsto-\left(y_{1}^{4}+y_{2}^{2}+\cdots\right.$ $\left.+y_{t-1}^{2}\right)$ where the first coordinate represents the direction of vanishing principal curvature. According to Bröcker (1975, 14.8 and 14.10) any corresponding l-parametric versal unfolding of $\left.u\right|_{\hat{p}, \tilde{w}}$ is the sum of a two-parametric universal unfolding $\left(y_{1}, \ldots, y_{1-1}, v_{1}, v_{2}\right) \mapsto-\left(y_{1}^{4}+v_{2} y_{1}^{2}\right.$ $\left.+v_{1} y_{1}+y_{2}^{2}+\cdots+y_{l-1}^{2}\right)$ and an (l-2)-parametric constant unfolding $\left(y_{1}, \ldots, y_{l-1}, v_{3}, \ldots, v_{l}\right) \mapsto-\left(y_{1}^{4}+y_{2}^{2}+\cdots+y_{l-1}^{2}\right)$. An l-parametric versal unfolding hence is given by

$$
\left(y_{1}, \ldots, y_{l-1}, v_{1}, v_{2}, \ldots, v_{l}\right) \stackrel{f}{\rightarrow}-\left(y_{1}^{4}+v_{2} y_{1}^{2}+v_{1} y_{1}+y_{2}^{2}+\cdots+y_{l-1}^{2}\right) .
$$

We shall assume that the unfolding $U$ of $\left.u\right|_{\vec{p} . \dot{w}}$ is equivalent to $f$ [see definition 14.2 in Bröcker (1975, p. 121)]. We shall show that this assumption together with the rank condition $\pi 3$ on $g$ ensures that the phenomenon of vanishing Gaussian curvature does not destroy differentiability of mean demand. Note that it is not necessary in this context to vary preferences. It suffices to aggregate with respect to wealth keeping the preference relation fixed.

Theorem 2. Let $\lesssim \in \mathscr{P}$ and assume $\mathscr{M}$. Suppose $\varphi_{\lesssim}(\tilde{p}, \tilde{w})=\{\tilde{x}\}$ and $\kappa(\tilde{x})=0$, Furthermore assume that the unfolding $U$ of $\left.u\right|_{\hat{p}, \hat{w}}$ is equivalent to $f$ which is defined by $f\left(y_{1}, \ldots y_{l-1}, v_{1}, v_{2}, \ldots, v_{l}\right)=-\left(y_{1}^{4}+v_{2} y_{1}^{2}+v_{1} y_{1}+y_{2}^{2}+\cdots+y_{l-1}^{2}\right)$. Then there is an $\varepsilon>0$ such that

$$
\int_{\dot{w}-\varepsilon}^{\dot{w}+\varepsilon} \varphi_{\leqslant}(\cdot, w) h_{\lessgtr}(w) \mathrm{d} w \text { is } \quad C^{1} \text { at } \tilde{p}
$$

The assumptior that $\varphi_{\leqslant}$is single-valued at $(\tilde{p}, \tilde{w})$ is made to exclude disturbing phenomena that might occur in addition to $k(\tilde{x})=0$. For a similar reason we reed the $\varepsilon$ in the conclusion of the theorem. Again, dropping the continuous density $h_{\S}$ will not influence the arguments.

Before we start proving the theorem we shall discuss the idea behind it. For this we introduce some of the notions of catastrophe theory which will play an important role in the proof.

Let us assume $l=2$ for a moment. Moreover think of the parameters $v_{1}, v_{2}$ in the two-parametric Iniversal unfolding $\left(y_{1}, v_{1}, v_{2}\right) \stackrel{f}{\rightarrow}-\left(y_{1}^{4}+v_{2} y_{1}^{2}+v_{1} y_{1}\right)$ as being wealth and price, respectively.

Then the singular locus of $f$ is defined by $\Sigma_{f}=\left\{\left(y_{1}, v_{i}, v_{2}\right) \in \boldsymbol{R}^{1} \times \boldsymbol{R}^{2} \mid y_{1}\right.$ is a critical point of $\left.f\left(\cdot, v_{1}, v_{2}\right)\right\}$. It is a smooth $l$-dimensional manifold. The catastrophe map projects $\Sigma_{f}$ to the $\left(v_{1}, v_{2}\right)$-space.

The set of parameters $v_{1}, v_{2}$ for which $f\left(\cdot, v_{1}, v_{2}\right)$ has at least one degenerate critical point is the catastrophe set, in our case the cusp. The 
Maxwell set $\left\{\left(v_{1}, v_{2}\right) \mid v_{1}=0, v_{2}<0\right\}$ is the set of parameters where there are two equal minima of the potential function $f\left(\cdot, v_{1}, v_{2}\right)$. The Maxwell convention asserts that, when the potential function $f\left(\cdot, v_{1}, v_{2}\right)$ has more than one local minimum, then the chosen state is the one of absolute minimum potential. Therefore the Maxwell set and the Maxwell convention play an important role, since they describe the situations at which a jump in demand occurs.

Outside $v_{1}=0$ we are off the Maxwell set. Hence there exists, for each $\left(v_{1}, v_{2}\right), v_{1} \neq 0$, a unique absolute minimum, $y_{1}\left(v_{1}, v_{2}\right)$, of the potential function $f\left(\cdot, v_{1}, v_{2}\right)$. This minimum represents the demand at price $v_{2}$ and wealth $v_{1}$. We want to show that

$$
r_{2} \mapsto \int_{-\varepsilon}^{0} y_{1}\left(v_{1}, v_{2}\right) \mathrm{d} v_{1}+\int_{0}^{+\varepsilon} y_{1}\left(v_{1}, v_{2}\right) \mathrm{d} r_{1}=\int_{-\varepsilon}^{+\varepsilon} y_{1}\left(v_{1}, v_{2}\right) \mathrm{d} r_{1},
$$

i.e., the mean demand, is $C^{1}$ at $v_{2}=0$.

We have to show that each of the two integrals on the right-hand side is $C^{1}$ at $v_{2}=0$. The difficulty is that the derivatives of the integrands, though existing everywhere except for the point $(0,0)$, do not stay bounded when the arguments approach $(0,0)$. So one has to show that the arguments $v_{1}, v_{2}$ tend to zero 'faster' than the derivatives of the integrands tend to infinity. For this one shows that the derivative of $v_{2} \mapsto \int_{-\varepsilon}^{0} y_{1}\left(v_{1}, v_{2}\right) \mathrm{d} v_{1}$ is the uniform limit of the derivatives of $v_{2} \mapsto \int_{-}^{-} \| y_{1}\left(v_{1}, v_{2}\right) \mathrm{d} v_{1}$ for $\eta$ tending to zero.

In the following proof of Theorem 2 we shall show that the identification of the parameters $v_{1}, v_{2}$ and the wealth price situations is qualitatively correct near the critical point, and we shall outline the computations yielding the differentiability of mean demand.

\section{Proof of Theorem 2}

Let $f$ be the unfolding defined in the theorem which is by assumption equivalent to $U$. Once $\hat{x}=\left(x_{1}, \ldots, x_{l-1}\right)$ is associated with $x=\left(x_{1}, \ldots, x_{l}\right)$ the equivalence of $f$ and $U$ establishes the following commutative diagram:

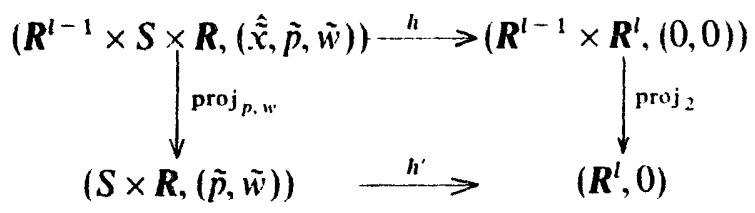


Here $h$ and $h^{\prime}$ are germs of diffeomorphisms. For any $(\hat{x}, p, w) \in \boldsymbol{R}^{l-1} \times S \times \boldsymbol{R}$ near $(\hat{\tilde{x}}, \tilde{p}, \tilde{w})$ we have

$$
\begin{aligned}
h(\hat{x}, p, w) & =\left(h_{1}(\hat{x}, p, w), \ldots, h_{l-1}(\hat{x}, p, w), h_{l}(\hat{x}, p, w), \ldots, h_{2 l-1}(\hat{x}, p, w)\right) \\
& =\left(h_{1}(\hat{x}, p, w), \ldots, h_{l-1}(\hat{x}, p, w), h_{1}^{\prime}(p, w), \ldots, h_{l}^{\prime}(p, w)\right) .
\end{aligned}
$$

We notice that the consumption set $X$ is $C^{x}$ diffeomorphic to its image in the singular locus $\Sigma_{v}$ of $U$ by the map $x_{\mapsto}{ }^{\dot{j}}(\hat{x}, g(x), g(x) x)$. The inverse of this map is given by $(\hat{x}, p, w)^{i-1}\left(\hat{x},(w-\hat{p} \hat{x}) \cdot\left(1-\sum_{h=1}^{l-1} p_{h}\right)^{-1}\right)$. The sets $\Sigma_{U}$ and $\Sigma_{f}$ $=\left\{\left(y_{1}, \ldots, y_{l-1}, v_{1}, \ldots, v_{l}\right) \in \boldsymbol{R}^{l-1} \times \boldsymbol{R}^{l} \mid v_{1}+2 v_{2} v_{1}+4 y_{1}^{3}=0, \quad y_{2}=\cdots=y_{l-1}=0\right\}$ are smooth $/$-dimensional manifolds.

If we clenote their germs at $(\hat{\tilde{x}}, \tilde{p}, w)$ resp. $(0,0,0)$ by the same symbols we have $\Sigma_{l^{\prime}}=h^{-1}\left(\Sigma_{f}\right)$. Computation of the kernel of the derivative of $\left(y_{1}, v_{1}, v_{2} \mapsto 4 y_{1}^{3}+2 v_{2} y_{1}+v_{1}\right.$ at $(0,0,0)$ shows that the tangent space $T_{(0,0)} \Sigma_{f}$ $\subset \boldsymbol{R}^{l-1} \times \boldsymbol{R}^{l}$ is the $l$-dimensional subspace with coordinates $y_{1}, v_{2}, \ldots, v_{l}$. Denote ty $M_{f}$ the closure of the Maxwell set $\left\{\left(v_{1}, \ldots, v_{l}\right) \mid v_{1}=0, v_{2}<0\right\}$ in the parameter space $\boldsymbol{R}$, i.e., $M_{f}=\left\{\left(v_{1}, \ldots, v_{l}\right) \mid v_{1}=0, v_{2} \leqq 0\right\}$. Analogously, $M_{U}$ denotes the closure of the Maxwell set of $U$. If we project $T_{(0.0)} \Sigma_{f}$ to the parameter space $R^{l}$ we just obtain the subspace spanned by the set $M_{f}$. Indeed,

$$
\operatorname{proj}_{2}\left(T_{(0.0,} \Sigma_{f}\right)=\left\{\left(v_{1}, \ldots, v_{l}\right) \mid v_{1}=0\right\}=T_{0} M_{f}
$$

By the equivalence of the unfoldings $U$ and $f$ we get (again denoting germs by the same symbols as their representatives)

$$
\begin{aligned}
& \operatorname{proj}_{p, w}\left(\Sigma_{U}\right)=h^{\prime-1}\left(\operatorname{proj}_{2}\left(\Sigma_{f}\right)\right), \\
& M_{U}=h^{\prime-1}\left(M_{f}\right)
\end{aligned}
$$

and

$$
\operatorname{proj}_{\rho, w}\left(T_{(\hat{\bar{x}}, \tilde{p}, \dot{w})} \Sigma_{U}\right)=T_{(\bar{p}, \tilde{w} ;} M_{U}
$$

Consider the following diagram:

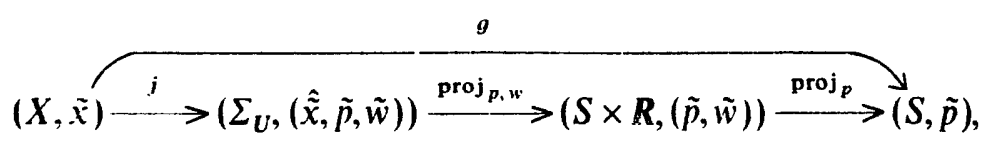

where $;$ is defined by $x \stackrel{j}{\mapsto}(\hat{x}, g(x), g(x) x)$. 
Using $\operatorname{proj}_{p, w}\left(T_{(\hat{\hat{x}, j i, i})} \Sigma_{U}\right)=T_{(\tilde{p}, \dot{w})} M_{U}$ we get

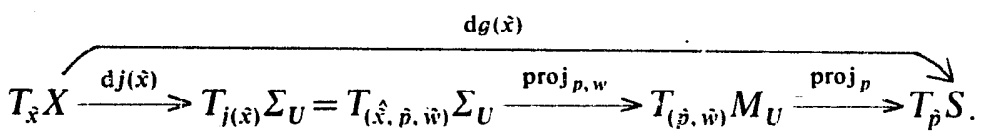

Since $g$ has full rank everywhere, the map $\operatorname{dg}(\tilde{x}): T_{\tilde{x}} X \rightarrow T_{\tilde{p}} S$ is surjective.

Thus $\operatorname{proj}_{p}: T_{(\bar{p}, \bar{w})} M_{U} \rightarrow T_{\dot{p}} S$ is surjective.

Since $\operatorname{dim} T_{(\bar{p}, \bar{w})} M_{U}=\operatorname{dim} T_{\hat{p}} S=l-1$ we conclude that $\operatorname{proj}_{\left.\right|_{M_{l}}}: M_{\ell} \rightarrow S$ defines a local diffeomorphism at $(\tilde{p}, \tilde{w})$.

Thus $\bar{w}=\operatorname{proj}_{w \mid M_{U}} \circ\left(\operatorname{proj}_{\left.p\right|_{M_{u}}}\right)^{-1}:(S, \bar{p}) \rightarrow(\boldsymbol{R}, \tilde{w})$ is a smooth germ. The graph of any representative of $\overline{\bar{w}}$ represents the germ of $M_{U}$ at $(\tilde{p}, \tilde{w})$. Now we choose a representative of $\overline{\bar{w}}$, also denoted $\overline{\bar{w}}$. Near $(\tilde{p}, \bar{w})$ the demand correspondence is single-valued except for the points $(p, \overline{\bar{w}}(p))$. For any $p$ near $\tilde{p}$ we can write the integral $\int_{\bar{w}(p)-\varepsilon}^{\bar{\omega}(p)+\varepsilon} \varphi(p, w) \mathrm{d} w$ of the correspondence $\varphi(p, \cdot)$ as a sum of two integrals of functions, i.e.,

$$
\int_{\bar{w}(p)-\varepsilon}^{\bar{w}(p)+\varepsilon} \varphi(p, w) \mathrm{d} w=\int_{\bar{w}(p)-\varepsilon}^{\overline{\bar{w}}(p)} \varphi^{l}(p, w) \mathrm{d} w^{\prime}+\int_{\bar{w}(p)}^{\bar{w}(p)+\varepsilon} \varphi^{u}(p, w) \mathrm{d} w .
$$

The graphs of the functions $\varphi^{\prime \prime}$ and $\varphi^{l}$ can be looked at as demand curves corresponding to curves in the upper and lower sheet of the singular locus $\Sigma_{\ell}$. [For a picture see, for example, Bröcker (1975, pp. 148, 156).]

It remains to show that the two right-hand integrals are $C^{1}$ at $\tilde{p}$. Since the proofs are analogous we shall prese this only for the first integral. That means we consider only parameters in the half space $\left\{\left(v_{1}, \ldots, v_{l} \mid v_{1} \leqq 0\right\}\right.$ and the corresponding parameters in the $(p, w)$-space. There are uniquely determined sheets of $\Sigma_{f}$ and $\Sigma_{U}$ respectively, corresponding to this half space of parameters. Expressed in terms of the unfolding $f$ we consider only points of $\Sigma_{f}$ belonging to the following set:

$$
\left\{\left(y_{1}, v_{1}, v_{2}\right) \in \Sigma_{f} \mid v_{1} \leqq 0, y_{1} \geqq 0\right\} .
$$

Define for any $p^{\prime \prime} \in S$ the set $E_{p^{\prime}}=\left\{(p, w) \in S \times \boldsymbol{R} \mid p=p^{\prime}\right\}$. Since $\operatorname{proj}_{p\}_{u^{\prime}}}: M_{\ell}$ $\rightarrow S$ is a local diffeomorphism at $(\tilde{p}, \tilde{w})$ we know that $E_{\dot{p}}$ 皮 $M_{U}$ at $(\tilde{p}, \tilde{w})$. By the equivalence of the unfoldings $U$ and $f$ this implies $h^{\prime}\left(E_{\dot{p}}\right)$ 雨 $M_{f}$ at 0 . Then for any $p^{\prime}$ near $\tilde{p}$ the set $h^{\prime}\left(E_{p^{\prime}}\right)$ intersects $M_{f}$ transversally. Therefore the projection from $R^{l}$ onto its $v_{1}$-axis, restricted to $h^{\prime}\left(E_{p^{\prime}}\right)$ and denoted $\pi_{p^{\prime}}$, defines a $C^{\infty}$ chart of $h^{\prime}\left(E_{p^{\prime}}\right)$ near $h^{\prime}\left(E_{p^{\prime}}\right) \cap M_{f^{\prime}}$. Consequently, the map $\pi_{p^{\prime}} \circ h^{\prime}$ from $E_{p^{\prime}}$ into the $v_{1}$-axis of $\boldsymbol{R}^{l}$ defines a $C^{*}$ chart near $E_{p^{\prime}} \cap M_{U^{\prime}}$. Thus $v_{1}$ is a local coordinate of $E_{p}$, based at $E_{p} \cap M_{U}=\left\{\left(p^{\prime}, \overline{\bar{w}}\left(p^{\prime}\right)\right)\right\}$.

Now, for any $p$ near $\tilde{p}$ the local inverse of $\pi_{p} \circ h^{\prime}$ associates with any $v_{1}$ near zero a point $h^{\prime-1} \circ \pi_{p}^{-1}\left(v_{1}\right)=\left(p, w_{p}\left(v_{1}\right)\right) \in E_{p}$. This defines a local $C^{x}$ 
diffeomorphism $w_{p}: \boldsymbol{R} \rightarrow \boldsymbol{R}$ near zero. Therefore, for any $(p, w)$ with $w$ near $i(p)$ we have $(p, w)=\left(p, w_{p}\left(v_{1}\right)\right)$ for some $v_{1}$ near zero. Clearly, $w_{p}\left(v_{1}\right)$ tends to $\bar{w}(p)$ for $v_{1}$ tending to zero.

Now we fix a sufficiently small compact neighborhood $\bar{V}$ of $\tilde{p}$. For any $\eta \in] 0, a[$, we define the mapping

$$
\boldsymbol{\Phi}_{\eta}: S \rightarrow \boldsymbol{R}_{+}^{l}: p \mapsto \int_{\dot{w}(p)-\varepsilon}^{\vec{w}(p)-\eta} \varphi^{l}(p, w) \mathrm{d} w .
$$

The mapping $\Phi_{\eta}$ is $C^{1}$ at $\tilde{p}$ since $\varphi^{l}, \bar{w}(\cdot)-\varepsilon$ and $\overline{\bar{w}}(\cdot)-\eta$ are. Its derivative at $\tilde{p}$ is

$$
D \Phi_{\eta}(\tilde{p})=\int_{\tilde{w}(\hat{p})-\varepsilon}^{\tilde{w}(\hat{p})-\eta} D_{p} \varphi^{l}(\tilde{p}, w) \mathrm{d} w^{\prime}+D \overline{\bar{w}}(\tilde{p})\left(\varphi^{l} \tilde{p}(\bar{w}(\tilde{p})-\eta)-\varphi^{l} \tilde{p} \bar{w}(\tilde{p})-\varepsilon\right) .
$$

Clearly, for $\eta$ tending to zero, $\Phi_{\eta}$ converges pointwise to $\Phi$, which is defined by

$$
\boldsymbol{\phi}: S \rightarrow \boldsymbol{R}_{+}^{l}: p \mapsto \int_{w=1}^{\tilde{w}(p)-\varepsilon} \varphi^{l}(p, w) \mathrm{d} w .
$$

Therefore, if $D \Phi_{\eta}$ converges uniformly in the compact neighborhood $\bar{V}$ of $\tilde{p}$ for $\eta$ tending to zero, we get by Lang (1969, theorem 12, p. 117):

(1) $\Phi_{\eta}$ converges to $\Phi$ uniformly on $\ddot{V}$ for $\eta$ tending to zero.

(2) $\Phi$ is $C^{1}$ at any $p \in \bar{V}$.

(3) $D \Phi(p)=\lim _{\eta \rightarrow 0} D \Phi_{\eta}(p)$ for any $p \in \hat{V}$.

Hence it remains to show that $D \Phi_{\eta}$ converges uniformly on $V$. For the second term of the sum this is an immediate consequence of the continuity of $\varphi^{\prime}$. Thus we have to show that

$$
\int_{n+1-\varepsilon}^{n+1} D_{p} \varphi^{l}(\cdot, w) \mathrm{d} w
$$

converges uniformly on $\bar{V}$ for $\eta$ tending to zero.

Equivalently, we show that

$$
\int_{n=1 \cdot-1-\eta}^{\bar{w}(\cdot)} D_{p} \varphi^{l}(\cdot, w) \mathrm{d} w
$$

tends to zero uniformly on $\bar{V}$. 
For this it is sufficient to prove the uniform convergence to zero on $V$ of

$$
\int_{\bar{w}(\cdot)-\eta}^{\bar{w}(\cdot)}\left\|D \varphi^{l}(\cdot, w)\right\| \mathrm{d} w .
$$

Now define the map $\psi: \boldsymbol{R}^{l} \rightarrow \Sigma_{f}$ by

$$
\psi:=h \circ j \circ \varphi^{l} \circ h^{\prime-1} \text {. }
$$

Since the mappings $h, j, h^{-1}, \pi_{p}^{-1}$ define local diffeomorphisms it suffices to prove that

$$
\begin{gathered}
w_{p}^{-1} \int_{\left.\left(w^{\prime}(p)-\eta\right)\right)}^{\left.w_{p}^{-1}(w)(p)\right)} \| D(h \circ j)\left(\varphi^{l}\left(p, w_{p}\left(v_{1}\right)\right)\right) \circ D \varphi^{l}\left(p, w_{p}\left(v_{1}\right)\right) \\
\circ D h^{-1}\left(\pi_{p}^{-1}\left(v_{1}\right)\right) \circ D \pi_{p}^{-1}\left(v_{1}\right) \| \mathrm{d} v_{1}
\end{gathered}
$$

converges to zero uniformly on $\bar{V}$. Remembering that $h^{\prime-1} \circ \pi_{p}^{-1}\left(v_{1}\right)$ $=\left(p, w_{p}\left(c_{1}\right)\right)$ and $\overline{\bar{w}}(p)=w_{p}(0)$, and abbreviating $w_{p}^{-1}\left(w_{p}(0)-\eta\right)$ by $\rho(p, \eta)$ we can write this last integral as

$$
\int_{\rho(p, \eta)}^{0}\left\|D \psi\left(h^{\prime}\left(p, w_{p}\left(v_{1}\right)\right)\right) \circ D \pi_{p}^{-1}\left(v_{1}\right)\right\| \mathrm{d} v_{1} .
$$

Since $\pi_{p}^{-1}$ is a local diffeomorphism it suffices to prove that

$$
\int_{\rho(p, \eta)}^{0}\left\|D \psi\left(h^{\prime}\left(p, w_{p}\left(v_{1}\right)\right)\right)\right\| \mathrm{d} v_{1}
$$

converges to zero uniformly on $\bar{V}$.

As we know that $\psi\left(h^{\prime}\left(p, w_{p}\left(v_{1}\right)\right)\right) \in \Sigma_{f}$ and that $y_{2}=\cdots=y_{!}=0$ for all $\left(y_{1}, \ldots, v_{l-1}, v_{1}, \ldots, v_{l}\right) \in \Sigma_{f}$, we get

$$
\begin{aligned}
\psi\left(h^{\prime}\left(p, w_{p}\left(v_{1}\right)\right)\right)= & \left(y_{1}\left(h^{\prime}\left(p, w_{p}\left(v_{1}\right)\right)\right), 0, \ldots, 0, h_{1}^{\prime}\left(p, w_{p}\left(v_{1}\right)\right), \ldots\right. \\
& \left.h_{l}^{\prime}\left(p, w_{p}\left(v_{1}\right)\right)\right)
\end{aligned}
$$

It suffices to show that

$$
\int_{\rho(p, \eta)}^{0} \| D y_{1}\left(h^{\prime}\left(p, w_{p}\left(v_{1}\right)\right)\right) \mathrm{d} v_{1} \text { and } \int_{\rho(p, \eta)}^{0}\left\|D h^{\prime}\left(p, w_{p}\left(v_{1}\right)\right)\right\| \mathrm{d} v_{1}
$$


converge to zero uniformly on $\bar{V}$. For the second integral this follows directly from the fact that $h^{\prime}$ is a local diffeomorphism.

By definition of $\Sigma_{f}$ we have for $i=3, \ldots, l$,

$$
\frac{\partial y_{1}}{\partial v_{i}}\left(h^{\prime}\left(p, w_{p}\left(v_{1}\right)\right)\right)=0
$$

For $i=1,2$, we have

$$
\left|\frac{\partial y_{1}}{\partial v_{i}}\left(h^{\prime}\left(p, w_{p}\left(v_{1}\right)\right)\right)\right| \leqq\left|\frac{\partial y_{1}}{\partial v_{i}}\left(v_{1}, 0, h_{3}^{\prime}\left(p, w_{p}\left(v_{1}\right)\right), \ldots, h_{l}^{\prime}\left(p, w_{p}\left(v_{1}\right)\right)\right)\right|
$$

Moreover we get

$$
\begin{aligned}
& \left|\frac{\partial y_{1}}{\partial v_{1}}\left(v_{1}, 0, v_{3}, \ldots, v_{l}\right)\right| \leqq \text { const } \cdot\left\|v_{1}^{-\frac{2}{3}}\right\|, \\
& \left|\frac{\partial y_{1}}{\partial v_{2}}\left(v_{1}, 0, v_{3}, \ldots, v_{l}\right)\right| \leqq \text { const } \cdot\left\|v_{1}^{-\frac{t}{3}}\right\| .
\end{aligned}
$$

Thus, for any $p \in \bar{V}$. we have

$$
\begin{aligned}
\int_{\rho(p, \eta)}^{0}\left\|D \psi\left(h^{\prime}\left(p, w_{p}\left(v_{1}\right)\right)\right)\right\| \mathrm{d} v_{1} & \leqq \text { const } \cdot\left|\int_{\rho(p, \eta)}^{0} v_{1}^{-\frac{2}{3}} \mathrm{~d} v_{1}\right| \\
& =\text { const } \cdot \rho^{\frac{1}{3}}(p, \eta) .
\end{aligned}
$$

Since $\bar{V}$ is compact and since $(\rho(\cdot, \eta))_{\eta \rightarrow 0}$ is a decreasing sequence of realvalued functions defined on $\bar{V}$ converging to zero, the sequence converges to zero uniformly on $\bar{V}$. Therefore

$$
\int_{w^{\prime}(p)-\eta}^{w^{\prime}(p)}\left\|D_{p} \varphi^{l}(p, w)\right\| \mathrm{d} w \leqq \text { const } \cdot \max _{p \in \bar{V}} \rho^{\frac{1}{3}}(p, \eta)
$$

which converges to zero for $\eta$ tending to zero. Hence $D \Phi_{\eta}(p)$ converges to $D \Phi(p)$ unifo, mly on $\bar{V}$. Q.E.D.

\section{References}

Araujo, A. and A. Mas-Colell, 1978, Notes on the smoothing of aggregate demand, Jcurnal of Mathematical Economics, forthcoming.

Bröcker, T., 1975, Differentiable germs and catastrophes, LMS Lecture Notes Series 17 (London Mathematical Society, Cambridge University Press, Cambridge).

Debre1, G., 1972, Smooth preferences, Econometrica 40, 603-615.

Dieudonné, J., 1960, Foundations of modern analysis (Academic Press, New York). 
Dierker, E., H. Dierker and W. Trockel, 1980, Continuous mean demand functions derived from nonconvex preferences, Journal of Mathematical Economics 7, 27-33.

Freedman, D., 1971, Markov chains (Holden-Day, San Francisco, CA).

Golubitsky, M. and V. Guillemin, 1973, Stable mappings and their singularities (Springer, Berlin).

Hildenbrand, W., 1974, Core and equilibria of a large economy (Princeton University Press, Princeton, NJ).

Lang, S., 1969, Real analysis (Addison-Wesley, London).

Sondermann, D., 1975, Smoothing demand by aggregation, Journal of Mathematical Economics 2, 201-223.

Sondermann, D.. 1976, On a measure theoretical problem in mathematical economies, in: Springer Lecture Notes 541 (Springer, Berlin).

Wassermann, G., 1974, Stability of unfoldings, Springer lecture notes 393 (Springer, Berlin). 